\title{
Exploiting the Oxygen Redox Reaction and Crystal-Preferred Orientation in a P3-Type $\mathrm{Na}_{2 / 3} \mathrm{Mg}_{1 / 3} \mathrm{Mn}_{2 / 3} \mathrm{O}_{2}$ Thin-Film Electrode
}

Yiman Zhang ${ }^{1}$, Nathan D. Phillip ${ }^{2}$, Bohang Song ${ }^{3}$, Robert L. Sacci ${ }^{1}$, Jue Liu ${ }^{3}$, Nancy J. Dudney $^{1 *}$

${ }^{1}$ Chemical Science Division, Oak Ridge National Laboratory, Oak Ridge, Tennessee 37831, United States

${ }^{2}$ The Bredesen Center for Interdisciplinary Research and Graduate Education, University of Tennessee, Knoxville, Tennessee 37996, United States

${ }^{3}$ Neutron Scattering Division, Oak Ridge National Laboratory, Oak Ridge, Tennessee 37831, United States

*E-mail: zhangy4@ornl.gov dudneynj@ornl.gov
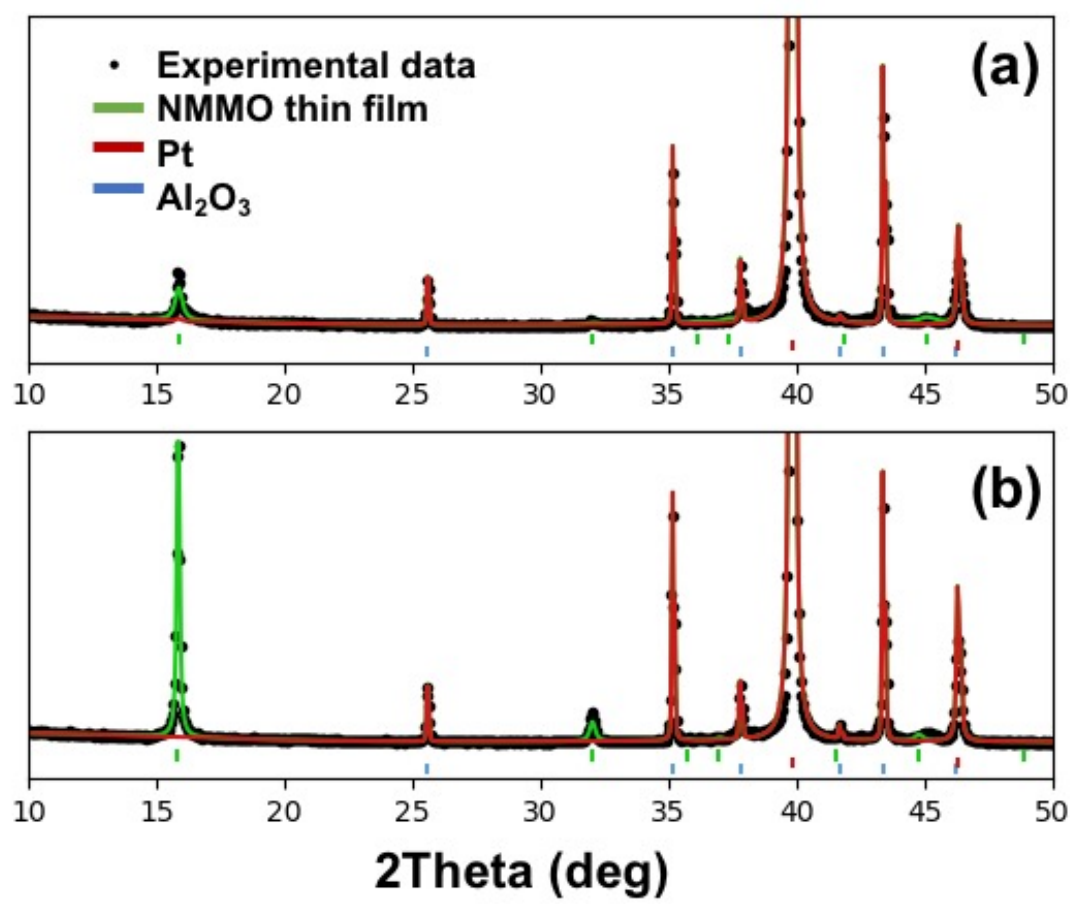

Figure S1. The visual fit for lab XRD pattern for both c-ROF NMMO thin film (a) and c-OF thin film (b). The intensity due to NMMO thin film diffraction is highlighted in green. 


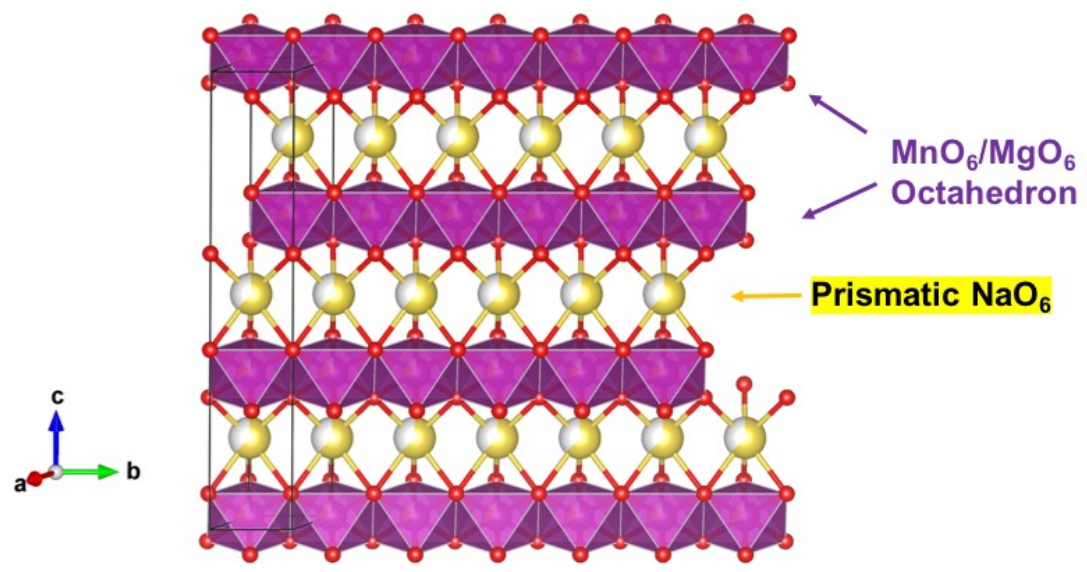

Figure S2. The R3 structure after fitting the diffraction of c-ROF. The graph is exported from the VESTA software

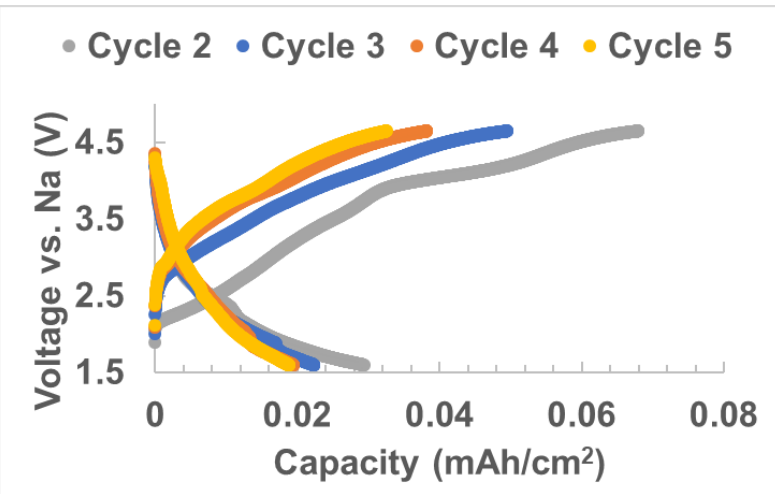

Figure S3. Galvanostatic cycling curves from cycle 2 to 5 for c-ROF electrode

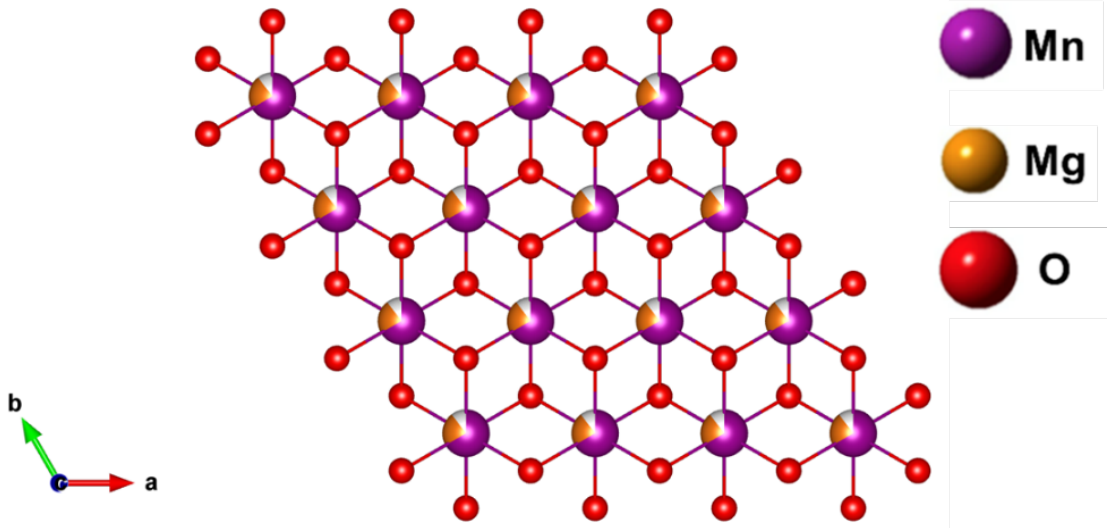

Figure S4. The top view of the (003) plane, which is a transition metal layer 